\title{
Factores Psicosociales y Síndrome de Burnout en Personal de Enfermería de una Unidad de Tercer Nivel de Atención a la Salud
}

\section{PSYCHOSOCIAL FACTORS AND BURNOUT SYNDROME IN NURSES OF A UNIT OF TERTIARY HEALTH CARE}

\author{
María Guadalupe Aldrete Rodríguez'1, Cristina Navarro Meza², Raquel González Baltazar1, Mónica I. Contreras Estrada, Jorge \\ Pérez Aldrete ${ }^{3}$ \\ 1. Instituto de Investigación en Salud Ocupacional Universidad de Guadalajara. \\ 2. Hospital Regional Valentín Gómez Farías ISSSTE. \\ 3. Maestria en Ciencias de la Salud en el Trabajo, Universidad de Guadalajara.
}

\begin{abstract}
RESUMEN
El objetivo fue determinar la relación entre los factores psicosociales laborables y el sindrome de Burnout en personal de enfermería de una unidad de tercer nivel de atención a la salud. Material y Métodos: Se trata de un estudio observacional, transversal y analítico en personal de enfermería de una unidad de tercer nivel de atención a la salud. Los datos se captaron con el instrumento de factores psicosociales de Silva y el MBI. Se analizaron con el apoyo del SPSS. Resultados: Participaron 163 personas de 21 servicios de la unidad de atención; 88\% eran mujeres, 41,4\% laboraba en el turno matutino y $47,5 \%$ perciben la presencia de factores psicosociales negativos en su área de trabajo, siendo lo más frecuente las exigencias laborales, remuneración del rendimiento y condiciones inadecuadas en su lugar de trabajo. En cuanto al Burnout, 33,3\% presentaba agotamiento emocional, 50,8\% baja realización personal y 17,9\% despersonalización. El agotamiento emocional se relacionó $(\mathrm{p}<\mathrm{a} 0,05)$ con seis de las ocho áreas de los factores psicosociales evaluados Conclusión: Se confirmó la relación entre los factores psicosociales laborales y el síndrome de Burnout.
\end{abstract}

(Aldrete M, Navarro C, González R, Contreras M, Pérez J, 2015. Factores Psicosociales y Síndrome de Burnout en Personal de Enfermería de una Unidad de Tercer Nivel de Atención a la Salud. Cienc Trab. Ene-Abr; 17 [52]: 32-36).

Palabras claves: FACTORES PSICOSOCIALES LABORALES, SÍNDROME DE BURNOUT, PERSONAL DE ENFERMERÍA.

\section{ABSTRACT}

The objective was to determine the relationship between psychosocial factors and working burnout syndrome in nursing staff of a unit of tertiary health care. Material and Methods: It is an observational, cross-sectional analytical study in nursing staff of a unit of tertiary health care. The data were collected with the instrument of psychosocial factors Silva and MBI. It was analyzed with the support of SPSS. Results: A total of 163 people from 21 services care unit; 88\% were women, 41,4\% worked in the morning shift and 47,5\% perceive the presence of negative psychosocial factors in their work area, where work demands are the most frequent, performance reward and inadequate conditions in work place. Regarding the Burnout, 33,3\% had emotional exhaustion, 50,8\% reduced personal accomplishment and depersonalization $17,9 \%$. Emotional exhaustion was associated $(p<0,05)$ with six of the eight areas of psychosocial factors evaluated Conclusion: the relationship between psychosocial factors and confirmed Burnout syndrome.

Key words: PSYCHOSOCIAL WORK FACTORS, BURNOUT SYNDROME, PERSONAL NURSING.

\section{INTRODUCCIÓN}

Los trabajadores de salud, por las características de su trabajo que incluye situaciones desgastantes a las que se enfrentan en sus actividades cotidianas, con altas exigencias y múltiples factores psicoso-

\section{Correspondencia / Correspondence:}

Dra. Maria Guadalupe Aldrete Rodríguez

Menhir No. 1130

Colonia Altamira Zapopan Jalisco

C.P. 45160

e-mail:draaldrete@yahoo.com.mx

Recibido: 20 de Septiembre de 2014 / Aceptado: 08 de Enero 2015 ciales, así como la transferencia que reciben de sus pacientes, constituyen un grupo de alta vulnerabilidad para desarrollar problemas relacionados con el estrés.

Organizaciones internacionales como la Organización Mundial de la Salud (OMS) y la Internacional del Trabajo (OIT), llaman la atención sobre aspectos psicosociales que afectan a los trabajadores de salud, tal es el caso de las directrices marco para combatir la violencia laboral en el sector salud ${ }^{1}$; mencionan que si bien la violencia laboral afecta a todos los sectores de trabajadores, uno de los sectores con más riesgo es el de la atención a la salud, puesto que de la violencia que se vive en el lugar de trabajo, el sector salud ocupa la cuarta parte, relacionándose con la tensión en el trabajo, la inestabilidad social, el deterioro de las relaciones interpersonales y otros factores psicosociales en el lugar de trabajo. Factores que en conjunto pueden estar presentes en más de la mitad de los trabajadores y en mayor presencia en las mujeres. 
Como consecuencias directas de la presencia de todos estos factores en los trabajadores del sector salud se tienen el deterioro de la calidad de los servicios de salud que se otorgan por estos trabajadores y el riesgo de abandono de sus profesiones por parte de los mismos, lo que reduciría los servicios de salud disponibles hacia la población general y aumentaría los costos de la salud. ${ }^{1}$ Desde hace décadas se ha reconocido que el trabajo de enfermería en el contexto hospitalario tiene varias fuentes de estrés y que las enfermeras al estar expuestas de manera constante, pueden tener repercusiones en su salud física y mental; sin embargo esto depende de diversos factores entre ellos los factores psicosociales que median la relación causa efecto ${ }^{2}$; tales como el trato con los usuarios demandantes, el contacto con la enfermedad, el dolor, la muerte, la escasez de personal, sobrecarga laboral y dobles jornadas de trabajo. ${ }^{3-6}$

El Comité Mixto de la Organización Internacional del Trabajo y la Organización Mundial de la Salud (OIT/OMS) definen a los factores psicosociales en el trabajo como "interacciones entre el trabajo, su medio ambiente, la satisfacción en el empleo y las condiciones de su organización, por una parte; y por la otra, las capacidades del empleado, sus necesidades, su cultura y su situación personal fuera del trabajo; todo lo cual a través de percepciones y experiencias influyen en la salud y el rendimiento".

Estudios más recientes definen los factores psicosociales como las condiciones presentes en situaciones laborales relacionadas con la organización del trabajo, el tipo de puesto, la realización de la tarea, incluso el entorno; que afectan el desarrollo del trabajo y la salud de las personas trabajadoras. Se ha demostrado que los factores psicosociales en el trabajo suelen tener un carácter crónico lo que supone una tensión psicológica continua para el individuo. ${ }^{8}$ Esta tensión crónica puede llevar a desencadenar algunos riesgos psicosociales como el síndrome de Burnout, que es considerado como resultante de una exposición crónica al estrés laboral.

El Síndrome de Burnout, es un sindrome psicológico que se presenta en respuesta a estresores crónicos presentes en el trabajo, generado en profesionales que mantienen una relación constante y directa con otras personas, primordialmente cuando ésta es de ayuda como en el caso de docentes, médicos, enfermeras, entre otros. Quien lo presenta, manifiesta actitudes y sentimientos negativos hacia las personas con las que se trabaja y hacia el propio rol profesional, así como por la vivencia de encontrarse emocionalmente agotado. ${ }^{9}$

Un estudio realizado con enfermeras de un centro de cancerología del estado de Durango, reporta un 3\% con agotamiento emocional en el nivel alto, 68\% con nivel bajo de realización personal y un $3 \%$ en el nivel medio de despersonalización. En relación a los factores psicosociales negativos presentes en el lugar de trabajo reportan un 20,3\% en el nivel alto. ${ }^{10}$

El estudio de los factores psicosociales laborales está en una etapa incipiente, dado que la mayor parte de los trabajos abordan los efectos de estos factores y no los factores psicosociales en sí. ${ }^{11}$

Hasta ahora, son pocos los estudios en nuestro país que demuestran la relación de los factores psicosociales laborales con el síndrome de Burnout sobretodo en poblaciones ocupacionales altamente vulnerables como es el personal de enfermería, el que se caracteriza por altos niveles de estrés como se constata en diversos estudios. ${ }^{12}$

Sobre la base de lo anteriormente expuesto se considera relevante explorar la presencia de factores psicosociales, así como del síndrome de Burnout en el personal de enfermería incluido en esta investigación.
El objetivo de la presente investigación fue determinar la existencia de una relación estadísticamente significativa entre los factores psicosociales laborables y el síndrome de Burnout en personal de enfermería de una unidad de tercer nivel de atención a la salud.

\section{MATERIAL Y MÉTODOS}

\section{Población de estudio}

Se trató de un estudio observacional, transversal y analítico autorizado por el comité de ética de la coordinación del área de enseñanza e investigación del hospital donde se realizó el estudio. La participación fue de manera anónima y otorgaron su consentimiento informado.

El personal de enfermería participante fue de 163, de diferentes puestos y departamentos de un hospital de tercer nivel del sector público, seleccionados mediante un muestreo probabilístico, no se incluyeron en el estudio aquellas que no cumplieron con los criterios de inclusión: antigüedad mínima de seis meses en el puesto de trabajo y aceptar participar.

\section{Instrumentos}

Para la recolección de datos se aplicó una batería de instrumentos compuesta por tres apartados. Uno de datos sociodemográficos y laborales; el segundo lo conformó la escala de Factores Psicosociales en el Trabajo de Silva (2006, modificado), estructurado en 7 subescalas: condiciones del lugar de trabajo, carga de trabajo, contenido y características de la tarea, exigencias laborales, papel del trabajador y desarrollo de la carrera, interacción social y aspectos organizacionales y por último, remuneración del rendimiento. Utiliza una escala de frecuencia de 5 grados, tipo Likert, que va de 0 (nunca) a 4 (siempre). Para su evaluación se suman los puntajes de cada apartado y se clasifican en tres categorias: bajo, medio y alto. Este instrumento tiene una confiabilidad promedio entre los factores con una alpha de cronbach de 0,8817 , por lo que podemos asumir que tiene una validez de constructo y confiabilidad interna aceptable. ${ }^{13}$

El tercer apartado incluyó la escala Maslash Bournout Inventory (M.B.I.), estructurada con respuestas de opción múltiple, tipo Likert con siete categorias que van desde "nunca" que tiene valor de cero hasta "todos los días" con valor de 6 . Es un cuestionario que contiene 22 ítems, organizado en tres subescalas: cansancio emocional CE (nueve ítems), despersonalización DP (cinco ítems), y falta de realización personal FRP (ocho items). ${ }^{14}$ Las puntuaciones de la escala se obtienen de sumar los valores por cada una de las subescalas. Bajas puntuaciones en falta de realización personal y altas puntuaciones en agotamiento emocional y en despersonalización suponen la presencia de desgaste profesional. ${ }^{15}$ Como punto de corte se utilizó la versión catalana del M.B.I. que maneja la clasificación como "alto", "medio" y "bajo". En cada una de las tres dimensiones se sumaron los niveles alto y medio para considerar el "daño". Se ha validado la confiabilidad del MBI valores alpha reportados en el manual técnico del MBI-HSS son 0,90 para agotamiento emocional, 0,79 para despersonalización y 0,71 para realización personal. ${ }^{15-17}$

\section{Análisis}

Para el análisis de resultados se utilizaron estadísticas descriptivas según el tipo de variables; para variables cualitativas frecuencias absolutas y relativas y para las variables cuantitativas medias y desviaciones estándar. 
Este análisis consideró los resultados de las siguientes variables: sociodemográficas y laborales, prevalencia del síndrome de Burnout y de cada una de sus dimensiones, factores psicosociales con los datos obtenidos en cada factor y en forma total.

Para el análisis inferencial y verificar la asociaciones entre las variables del estudio se utilizaron diferentes pruebas estadísticas Ji cuadrada de person con o sin corrección de Yates se consideró una $\mathrm{p}<$ a 0,05, OR mayor a uno e intervalo de confianza del 95\% que no incluyera la unidad. Para este análisis las variables se organizaron en tablas $2 \mathrm{X} 2$ de la siguiente manera: Si burnout calificaciones de alto y medio en cada una de las dimensiones, No Burnout calificaciones bajas en cada dimensión. Número de dimensiones afectadas: Ninguna una o más. Para los factores psicosociales en cada uno de los factores se agruparon los resultados alto y medio como si riesgo y bajo como no riesgo.

\section{RESULTADOS}

Los participantes en el estudio laboraban en 21 servicios de la unidad de atención, 88\% eran del sexo femenino, con un rango de edad de los 28 a los 65 años, con un promedio de 38,9 y una desviación estándar de 10,59. Con respecto al estado civil, el 48,2\% eran casados, seguido de los solteros con 31,5\%. En cuanto a la escolaridad, el 49,7\% tenía estudios técnicos y en segundo lugar lo conformaban quienes habían cursado la licenciatura $(23,3 \%)$.

En relación a las características laborales el 41,4\% trabajaba en el turno matutino y 34,0\% en el turno nocturno. Tenían una antigüedad en la institución en promedio de 14,6 años, solo el 19,1\% tenía otro empleo y la mayoría trabajando en el área de enfermería con un promedio de 21 horas a la semana.

En cuanto a los factores psicosociales en la calificación general del instrumento se encontró que el 47,5\% del personal de enfermería perciben la presencia de los factores psicosociales negativos en su área de trabajo en el nivel medio o alto contra 52,5\% que dicen no estar presentes.

En relación a las subescalas se identificó la presencia de factores de naturaleza psicosocial en los espacios laborales más frecuentes en el nivel alto en exigencias laborales (31,5\%), en remuneración del rendimiento $(14,8 \%)$ y en condiciones del lugar de trabajo $(7,4 \%)$. La subescala con menor número de reportes fue la de contenido y características de la tarea, Tabla 1.

Tabla 1.

Factores Psicosociales en el Trabajo en personal de enfermería de una unidad de tercer nivel de atención a la salud.

\begin{tabular}{|c|c|c|c|c|c|c|}
\hline \multirow{2}{*}{$\begin{array}{l}\text { SUB-ESCALA } \\
\text { Factores }\end{array}$} & \multicolumn{2}{|c|}{ BAJO } & \multicolumn{2}{|c|}{ MEDIO } & \multicolumn{2}{|c|}{ ALTO } \\
\hline & $\mathrm{Fc}$ & $\%$ & $\mathrm{Fc}$ & $\%$ & $\mathrm{Fc}$ & $\%$ \\
\hline $\begin{array}{l}\text { Condiciones del } \\
\text { lugar de trabajo }\end{array}$ & 67 & 41,4 & 83 & 51,2 & 12 & 7,4 \\
\hline Carga de trabajo & 119 & 73,5 & 41 & 25,3 & 2 & 1,2 \\
\hline $\begin{array}{l}\text { Contenido y caracteristicas } \\
\text { de la tarea }\end{array}$ & 114 & 70,4 & 47 & 29,0 & 1 & 0,6 \\
\hline Exigencias laborales & 33 & 20,4 & 78 & 48,1 & 51 & 31,5 \\
\hline $\begin{array}{l}\text { Papel del trabajador y } \\
\text { desarrollo de la carrera }\end{array}$ & 118 & 72,8 & 40 & 24,7 & 4 & 2,5 \\
\hline $\begin{array}{l}\text { Interacción social y } \\
\text { aspectos organizacionales }\end{array}$ & 125 & 77,2 & 30 & 18,5 & 7 & 4,3 \\
\hline $\begin{array}{l}\text { Remuneración del } \\
\text { rendimiento }\end{array}$ & 77 & 47,5 & 61 & 37,7 & 24 & 14.8 \\
\hline $\begin{array}{l}\text { Total de factores } \\
\text { psicosociales }\end{array}$ & 85 & 52,5 & 75 & 46,3 & 2 & 1,2 \\
\hline
\end{tabular}

Fuente: Encuesta. Fc = (Frecuencia).
Tabla 2.

Presencia del síndrome de Burnout por número de dimensiones afectadas en personal de enfermería de una unidad de tercer nivel de atención a la salud.

\begin{tabular}{|ccc|} 
Número de dimensiones & Frecuencia & Porcentaje \\
0 & 56 & 34,6 \\
1 & 63 & 38,9 \\
2 & 27 & 16,7 \\
3 & 16 & 9,9 \\
\hline Total & 162 & 100,0 \\
\hline
\end{tabular}

Fuente: Encuesta.

Tabla 3.

Presencia del síndrome de Burnout por dimensión en personal de enfermería de una unidad de tercer nivel de atención a la salud.

\begin{tabular}{lcccccc}
$\begin{array}{l}\text { Dimensiones del Síndrome } \\
\text { de Burnout }\end{array}$ & \multicolumn{2}{c}{ BAJO } & \multicolumn{2}{c}{ MEDI0 } & \multicolumn{2}{c}{ ALTO } \\
Agotamiento emocional & 108 & 66,7 & 33 & 20,3 & 21 & 13,0 \\
Despersonalización & 133 & 82,1 & 16 & 9,9 & 13 & 8,0 \\
Baja realización Personal & 215 & 65,2 & 46 & 13,9 & 69 & 20,9
\end{tabular}

Fuente: Encuesta. Fc = (Frecuencia).

En lo que respecta al síndrome de Burnout, se encontró que 65,4\% presentaban al menos una dimensión afectada, de ellos 9,9\% reportaron las tres dimensiones, para esto se consideró la calificación como alta y media en cada una de las dimensiones, Tabla 2. Se identificó un 33,3\% con agotamiento emocional, un 50,8\% en baja realización personal y un 17,9\%, con despersonalización, Tabla 3.

Para encontrar la relación entre los factores psicosociales y el sindrome de Burnout se agruparon los resultados obtenidos en ambas escalas en alto y medio como "si riesgo", contra el resultado de bajo como "no riesgo", considerando los factores psicosociales como variable independiente y al Síndrome de Burnout como variable dependiente. Se encontró relación entre los factores psicosociales y el agotamiento emocional en seis de las ocho subescalas que evalúa el instrumento presentando mayor riesgo cuando se percibe mala interacción social (O.R 5,85 I.C. al 95\% 2,46 a $14,05 \mathrm{p}=0,000$ ), seguido de la suma del total de factores (O.R. 3,70 I.C. al 95\% 1,74-7,94 P = 0,000). La falta de realización personal, se comportó como factor de riesgo cuando se percibe que no existe un buen desarrollo personal (0.R. 2.37 I.C. al 95\% 1,08-5,24 $\mathrm{P}=0,0174)$.

La despersonalización se relacionó con la suma total de los factores psicosociales (0.R. 2.46 I.C. al 95\% 0,98-6,24 $\mathrm{P}=0,0323$ ), con la carga de trabajo (0.R. 2,79 I.C. al 95\% 1,11-7,04 $\mathrm{P}=0,0385$ ), y con la interacción social (0.R. 2.51 I.C. al 95\% 0,09-6,53 $\mathrm{P}=0,0326)$ Tabla 4.

Al relacionar los factores psicosociales con una o más dimensiones afectadas del MBI se encontró relación con: No percibir factores psicosociales negativos (O.R. $=0,48$ I.C. al 95\% 0,23-0,99 $\mathrm{P}=0,0285)$, percibir un buen desarrollo del trabajador (0.R. $=0,27$ I.C. al 95\% 0,10-0,79 $\mathrm{P}=0,00229)$, y tener una buena interacción social (O.R. $=0,08$ I.C. al 95\% 0,01-35 $\mathrm{P}=0,000$ ), se comportaron como factores protectores.

\section{DISCUSIÓN}

De acuerdo a las características del muestreo utilizado, los resultados se pueden extrapolar a la población general de donde se obtuvo la muestra. 
Tabla 4.

Asociación entre los factores psicosociales y las dimensiones del Síndrome de Burnout en personal de enfermería de una unidad de tercer nivel de atención a la salud.

\begin{tabular}{|c|c|c|c|}
\hline SUBESCALAS & $\begin{array}{l}\text { AGOTAMIENTO } \\
\text { EMOCIONAL }\end{array}$ & $\begin{array}{c}\text { BAJA } \\
\text { REALIZACIÓN }\end{array}$ & DESPERSONALIZACIÓN \\
\hline $\begin{array}{l}\text { Condiciones del } \\
\text { lugar de trabajo }\end{array}$ & * & $*$ & * \\
\hline Carga de trabajo & $\begin{array}{c}\text { O.R. } 2,49 \\
\text { I.C. }(1,13-5,48) \\
\text { P } 0,01185\end{array}$ & * & $\begin{array}{c}0 . R .2,79 \\
\text { I.C. }(1,11-7,04) \\
\text { P } 0,0385\end{array}$ \\
\hline $\begin{array}{l}\text { Contenido y } \\
\text { caracteristicas } \\
\text { de la tarea }\end{array}$ & $\begin{array}{c}\text { O.R. } 2,17 \\
\text { I.C. }(1,014.66) \\
\text { P } 0,0285\end{array}$ & * & * \\
\hline $\begin{array}{l}\text { Exigencias } \\
\text { laborales }\end{array}$ & $\begin{array}{c}\text { O.R. } 2,67 \\
\text { I.C. }(0,95-7,87) \\
\text { P } 0,0385\end{array}$ & * & * \\
\hline $\begin{array}{l}\text { Papel del trabajador } \\
\text { y desarrollo de } \\
\text { la carrera }\end{array}$ & $\begin{array}{c}\text { O.R. } 2,69 \\
\text { I.C. }(1,23-5,90) \\
\text { P } 0,0059\end{array}$ & $\begin{array}{c}\text { O.R. } 2,37 \\
\text { I.C. }(1,08-5,24) \\
\text { P } 0,0174\end{array}$ & * \\
\hline $\begin{array}{l}\text { Interacción social } \\
\text { y aspectos } \\
\text { organizacionales }\end{array}$ & $\begin{array}{c}\text { O.R. } 5,85 \\
\text { I.C. }(2,46-14,05) \\
\text { P } 0,0000\end{array}$ & * & $\begin{array}{c}\text { O.R. } 2,51 \\
\text { I.C. }(0,96-6,53) \\
\text { P } 0,0326\end{array}$ \\
\hline $\begin{array}{l}\text { Remuneración } \\
\text { del rendimiento }\end{array}$ & * & * & * \\
\hline $\begin{array}{l}\text { Total de factores } \\
\text { psicosociales }\end{array}$ & $\begin{array}{c}\text { O.R. } 3,70 \\
\text { I.C. }(1,74-7,94) \\
\text { P } 0,000\end{array}$ & * & $\begin{array}{c}\text { 0.R. } 2,46 \\
\text { I.C. }(0,98-6,24) \\
\text { P } 0,0323\end{array}$ \\
\hline
\end{tabular}

Se encontró similitud en las características sociodemográficas, con estudios anteriores realizados en personal de enfermería, por ejemplo, pertenecer en su mayoría al género femenino. ${ }^{10,18}$

En cuanto a los factores psicosociales, Gallegos et al. ${ }^{10}$ estudiaron cuatro áreas: dependiente de la tarea, sistema de trabajo, interacción social y relacionado con la organización misma y reportan que el 20,3\% fueron evaluados como altos y la mayoría considera que los factores estudiados no están presentes, situación contraria en el presente estudio en el que el 47,5\% de la población estudiada percibe niveles medios y altos de los factores estudiados. Por otra parte los hallazgos del presente estudio permiten confirmar la relación entre los factores psicosociales laborales y el síndrome de Burnout.

Flynn et al. El año $2009^{19}$, demostraron que altas cargas de trabajo y la falta de apoyo en el ambiente de trabajo, traen como consecuencia un deterioro de la calidad de atención y de la satisfacción del usuario, y esto se asociaba con un aumento significativo del síndrome de Burnout en las enfermeras; coincidiendo con el presente estudio, en el cual se demostró un mayor riesgo de presentar Burnout cuando se percibe una sobrecarga laboral y falta de interacción social.

Rodríguez et al. ${ }^{3}$, también reportan que el síndrome de Burnout está presente en las personas con deficientes relaciones sociales en el ámbito laboral.

En un estudio realizado en personal de enfermería de servicios de urgencia se reportó un 26\% con desgaste profesional. ${ }^{20}$ Por su parte, otro estudio realizado en el Instituto Nacional de Cancerología de la Ciudad de México en una población de enfermeras, reporta que 38\% de la población estudiada tenía el síndrome de Burnout y las autoras consideraban que el 59\% se encontraban en riesgo de padecerlo ${ }^{4}$, datos inferiores a los del presente estudio en el cual el $65,4 \%$ presentó al menos una dimensión afectada. Reyes en $2007^{21}$ reporta datos muy semejantes, el 63\% del personal de enfermería estudiado se encontraban afectados por el sindrome, por otra parte Rodríguez $^{3}$ reporta que el $88 \%$ de la población presenta el sindrome de Burnout en el nivel alto, por lo que esta población puede ser considerada como de alto riesgo y como un problema de salud pública, pues es una patología que se expresa en mayor medida en los profesionales del sector servicios que desarrollan su actividad laboral hacia otras personas ${ }^{22}$ y que puede tener otras implicaciones por la forma en que esta población brinda sus servicios hacia los pacientes reflejada en una deshumanización al brindar los cuidados de enfermería, apatía y actitudes negativas hacia el trabajo. ${ }^{3}$ En el presente estudio una de cada 3 personas presentó agotamiento emocional, esto pudiera derivar en una atención distante y fría con los usuarios, 17,9\% presentaron despersonalización y 34,8\% presentaron baja realización personal.

Los hallazgos del presente estudio permitieron confirmar la relación entre los factores psicosociales laborales y el sindrome de Burnout, por lo que las acciones deben ir más allá del ámbito personal y trabajar desde la organización con la modificación de ciertos factores que permitan una mayor interacción y apoyo social, así como un mejor desarrollo profesional ya que estos se presentaron como variables protectora.

El síndrome de Burnout tiene consecuencias en la esfera personal, en el ámbito laboral y familiar; al encontrar que 6 de cada 10 personas dedicadas al ámbito de la enfermería están afectadas por el síndrome, por esta razón se hace necesaria la implementación de programas de prevención y el diseño de estrategias para el manejo adecuado del estrés y la detección temprana de posibles casos. Además sería importante incorporar en la currículum de formación de este personal, esta temática para su prevención. 


\section{REFERENCIAS}

1. OIT-OMS.Organización Internacional del Trabajo-Organización Mundial de la Salud. Directrices marco para afrontar la violencia laboral en el sector salud[en línea]. Ginebra: OIT-OMS; 2002 [citado mar 2015].Disponible en:http://www. ilo.org/wcmsp5/groups/public/---ed_dialogue/---sector/documents/publication/wcms_160911.pdf

2. Fernández Garcia MV, Zárate Grajales RA, Bautista Paredes L, Domínguez Sánchez P, Ortega Vargas $C$, Cruz Corchado $M$, et al. Síndrome de Burnout y enfermeria. Rev Enferm Inst Mex Seguro Soc. 2012; 20(1):45-53.

3. Rodriguez Garcia $C$, Oviedo Zúñiga $A$, Vargas Santillán $M$, Hernández Velázquez V, Pérez Fiesco M. Prevalencia del Síndrome de Burnout en el personal de Enfermeria de dos Hospitales del Estado de México. Fundamentos en Humanidades. 2009; 19(1):179-193.

4. Gutiérrez González N, Pedraza Andrade C. Síndrome de desgaste profesional en el personal de enfermería de Instituto Nacional de Cancerologia Ciudad de México. Cancerología 2010; 5:31-35.

5. Zavala-González $M$, Posada-Arévalo $S$, Jiménez-Mayo 0 , López-Méndez $R$, Pedrero-Ramírez $L$, Pérez-Arias M. Síndrome de Burnout en personal médico y de enfermería de una unidad médica familiar en Tabasco, México. Rev. Med UV [en línea] 2011 [citado ene 2015]; 11(2):18-23. Disponible en: http://www.uv. $\mathrm{mx} / \mathrm{rm} /$ num_anteriores/revmedica_vol11_num2/articulos/sindrome.pdf.

6. Barrios Araya S, Arechabala Mantuliz Ma, Valenzuela Parada V. Relación entre carga laboral y Burnout en enfermeras de unidades de diálisis. Enferm Nefrol. 2012; 15(1):46-55.

7. OIT-OMS. Organización Internacional del Trabajo-Organización Mundial de la Salud. Factores psicosociales en el trabajo: naturaleza, incidencia y prevención. Informe del comité mixto OIT/OMS sobre medicina del trabajo. Ginebra: OIT, 1984. Disponible en http://biblioteca.uces.edu.ar/MEDIA/EDOCS/FACTORES_ Texto.pdf.

8. Gil-Monte PR. Riesgos psicosociales en el trabajo y salud ocupacional. Rev Perú Med Exp Salud Pública. 2012; 19(2):237-241.

9. Martínez Pérez A. El síndrome de Burnout. Evolución conceptual y estado actual de la cuestión. Vivat Academia [en línea] 2010 [citado ene 2015];112. Disponible en: http://ww w.ucm.es/info/vivataca/numeros/n112/DATOSS.htm. Maslach C, Jackson SE. MBI. Inventario Burnout de Maslach. Sindrome del "quemado" por estrés laboral asistencial. Madrid: TEA Edics.; 1997. (Publicaciones de Psicología Aplicada; 211).

10. Gallegos Alvarado M, Parra Domínguez $M$, Castillo Díaz R, Murillo Ortiz E. Sindrome de Burnout y factores psicosociales del personal de enfermeria al cuidado del paciente oncológico. CONAMED 2009;14:28-33.
11. Feldman $L$, Blanco $G$. Una aproximación al estudio de los factores psicosociales laborales en Venezuela. Salud de los Trabajadores [en línea]. 2012 [citado ene 2015]; 20(1):73-88. Disponible en:http://www.scielo.org.ve/scielo.php?script=s ci_arttext\&pid=S131501382012000100007\&lng=es\&nrm=iso

12. Juárez-García A. Factores psicosociales laborales relacionados con la tensión arterial y síntomas cardiovasculares en personal de enfermeria en México. Salud Pública Mex[en linea] 2007 [citado ene 2015]; 49(2):109-117. Disponible en: http://www.scielo.org.mx/scielo.php?script=sci_arttext\&pid=S0036-634 $2007000200006 \& \operatorname{lng}=e s \&$ tlng=es

13. Silva N. Factores psicosociales, estrés y desgaste profesional en académicos del Centro Universitario de Ciencias Biológicas Agropecuarias de la Universidad de Guadalajara [tesis de doctorado]. México: Universidad de Guadalajara; 2006.

14. Maslach C, Jackson SE, Leiter, MP. Maslach Burnout Inventory Manual. 3th ed. Palo Alto: Consulting Psychologists Press; 1996.

15. Meda R, Moreno B, Rodríguez A, Morante M, Ortiz G. Análisis factorial confirmatorio del MBI-HSS en una muestra de psicólogos mexicanos. Psicol Salud.2008; 18(1):107-116.

16. Gil Monte PR, Peiró JM. Validez factorial del Maslach Burnout Inventory en una muestra multiocupacional. Psicothema. 1999; 11(3):679-689.

17. Gil-Monte PR, Peiró JM. Un estudio comparativo sobre criterios normativos y diferenciales para el diagnóstico del Síndrome de Quemarse por el trabajo (Burnout) según el MBI-HSS en España. Rev Psicol Trab Organ. 2000; 16(2):135-149.

18. Álvarez Verdugo L, Prieto Bocanegra B. Prevalencia de desgaste profesional en personal de enfermería de un hospital de tercer nivel de Boyacá, Colombia. Enferm Glob. 2013; 19:73-88.

19. Flynn L, Thomas-Hawkins C, Clarke SP. Organizational traits, care processes and Burnout among chronic hemodialysis nurses. Western J Nurs Res. 2009; 31(5):569.

20, Cogollo-Milanés Z, Batista E, Cantillo Ch, Jaramillo A, Rodelo D, Meriño G. Desgaste profesional y factores asociados en personal de enfermería de servicios de urgencias de Cartagena Colombia. Aquichan. 2010; 10(1):43-51.

21. Reyes J, Soloaga G, Quintana P, Dos Santos L. El síndrome de Burnout en personal médico y de enfermería del Hospital "Juan Ramón Gómez" de la localidad de Santa Lucía, Corrientes. Argentina. Revista de Post-grado de la Via Cátedra de Medicina [en línea] 2007 [citado ene 2015]; 167:1-7. Disponible en: http://www.med.unne. edu.ar/revista/revista167/1_167.

22. Gil Monte PR. Algunas razones para considerar los riesgos psicosociales en el trabajo y sus consecuencias en la salud pública. Rev Esp Salud Pública. 2009; 83:169-173. 